\title{
LA GRAN PROSA: MERLEAU-PONTY Y LA LITERATURA COMO EXPRESIÓN DE LA VERDAD
}

\author{
Martín Buceta* \\ doi: 10.11144/Javeriana.uph37-75.gpmp
}

\section{RESUMEN}

El objetivo del artículo es revisitar el concepto de gran prosa elaborado por Merleau-Ponty para señalar la importancia del lenguaje literario en el problema de la expresión de la verdad. Para ello, expondremos las tesis principales del curso dictado por Merleau-Ponty en el año académico 1953-1954, titulado Le problème de la parole. Esto nos permitirá caracterizar al lenguaje literario y su capacidad de llevar a la expresión el sentido de la percepción. Por último, se considerará la necesidad de la incorporación de la expresión literaria en la elaboración de una filosofía de lo sensible.

Palabras clave: literatura; gran prosa; verdad; expresión

Universidad Católica Argentina - ConicET, Buenos Aires, Argentina.

Correo electrónico: tinbuceta@hotmail.com

Para citar este artículo: Buceta, M. (2020). La gran prosa: Merleau-Ponty y la literatura como expresión de la verdad. Universitas Philosophica, 37(75), 73-99. ISSN 0120-5323, ISSN en línea 2346-2426. doi: 10.11144/Javeriana.uph37-75.gpmp 


\title{
THE GREAT PROSE: MERLEAU-PONTY AND LITERATURE AS EXPRESSION OF TRUTH
}

\begin{abstract}
This article revisits the concept of great prose originally proposed by Merleau-Ponty to stress the importance of literary language in the problem of the expression of truth. To achieve this objective, the main theses of the course given by Merleau-Ponty in the 1953-1954 academic year, titled Le problème de la parole, will be discussed. A characterization of literary language and its ability to express the meaning of perception will follow. Finally, the need to incorporate literary expression in the elaboration of a philosophy of the sensible will be considered.
\end{abstract}

Keywords: literature; great prose; truth; expression 


\section{Introducción}

LA LITERATURA y su particular modo de expresión inquietó el pensamiento de Merleau-Ponty a lo largo de toda su obra. Es significativo que, ya desde el prólogo de Fenomenología de la percepción (1945), el filósofo emparente a la fenomenología con la literatura, en tanto que ambas "comparten el mismo género de atención y asombro, la misma exigencia de conciencia y la misma voluntad de captar el sentido del mundo o de la historia en estado naciente" (p. xvi). Sin embargo, durante un período particular de su producción ese parentesco se torna cada vez más significativo, hasta convertirse en un tema de reflexión principal de su tarea filosófica.

Entre 1951 y 1952, Merleau-Ponty emprende el inacabado ensayo en que interroga los trabajos del pintor y el escritor y presta especial atención al problema de la expresión, manuscrito que más adelante Claude Lefort titulará para su publicación La prosa del mundo [La prose du monde]. En el año académico siguiente, 1952-1953, Merleau-Ponty titula el curso de los jueves en el Collège de France El mundo sensible y el mundo de la expresión [Le monde sensible et le monde de l'expression]. Allí emprende un camino de renovación de las categorías con las que se piensa el mundo sensible e intenta redefinir ciertos conceptos fenomenológicos centrales para poder comprender el problema esencial de la filosofía. Este problema no es otro que aquel que había señalado Edmund Husserl y que es constantemente retomado por Merleau-Ponty: el problema que concierne a "la experiencia pura y, por así decirlo, aún muda, que debe ser llevada a la expresión pura de su propio sentido" (Husserl, 1950, p. 77)1'. No es casual que ese mismo año dedique el curso de los lunes a realizar una reflexión sobre el lenguaje

1 Es preciso aclarar que en todos los casos en que se ha citado una obra de edición francesa, inglesa o alemana la traducción es nuestra. Por ello, siempre se indicará el texto original en nota al pie entre corchetes. [„Der Anfang ist die reine und sozusagen noch stumme Erfahrung, die nun erst zur reinen Aussprache ibres eigenen Sinnes zu bringen ist"]. Respecto de esta nota en particular, debe aclararse que la inspiración que Merleau-Ponty encuentra en el pensamiento de Husserl deriva en una resolución del problema distinta. Merleau-Ponty -contrariamente a Husserl- propondrá que la expresión del sentido mudo de la percepción ha de darse no a través de un "lenguaje puro" propio de una conciencia constituyente que elaboraría aquella gramática universal del ser, sino por medio de un lenguaje que se hace en medio del ser y que recoge en sus pliegues la contingencia y la opacidad de la situación que lo ha instituido, un lenguaje que está envuelto en la carne del mundo y que, 
literario, titulándolo Investigaciones sobre el uso literario del lenguaje [Recherches sur l'usage littéraire du langage]. Es sugerente pensar, al menos en un primer acercamiento, que la clave para llevar a la expresión el mundo sensible puede hallarse en un determinado uso literario del lenguaje.

Por último, este período debe ser complementado con un curso inédito del año siguiente titulado: El problema de la palabra [Le problème de la parole]. El resumen de este curso nos adelanta la intención que Merleau-Ponty tiene de desentrañar el fenómeno del lenguaje literario para demostrar su función conquistadora y hacernos ver cómo el escritor "trata de producir un sistema de signos que restituya, gracias a su ordenamiento interno, el paisaje de una experiencia" (Merleau-Ponty, 1968, p. 40). Una de las ideas centrales que Merleau-Ponty (1953-1954) fundamenta en ese curso queda claramente expresada en la afirmación: "la literatura tiene por función conquistar una verdad" (p. 119v)².

La apelación merleaupontiana al lenguaje literario busca encontrar una vía de resolución al mentado problema de la expresión. La dificultad que se presenta en torno al fenómeno de la expresión -y que hemos indagado en trabajos previos (Buceta, 2017) - es la suscitada en torno a la inexplicada migración del sentido mudo de la percepción hacia el sentido proferido. Merleau-Ponty considerará que esta explicación debe hallarse en un determinado uso creativo del lenguaje propio del escritor- que permite sublimar en las palabras instituyentes el sentido perceptivo ${ }^{3}$.

siendo parte de la carne de lo visible, se prolonga en lo invisible. Para ahondar en la apropiación merleaupontiana de esta frase de Husserl, véase Waldenfels, 1998, pp. 331-348.

2 «la littérature a pour fonction de conquérir une vérité. » Para referir al curso solo recientemente publicado indicaremos la paginación del manuscrito inédito de la Biblioteca Nacional de Francia, agregando $v$ para "verso" en caso de que sea el reverso de la página.

3 En La prosa del mundo, Merleau-Ponty (1969) se refería a esta capacidad del lenguaje literario cuando afirmaba que "el lenguaje no dice nunca nada, lo que hace es inventar una gama de gestos que presentan entre sí diferencias lo suficientemente claras como para que el comportamiento del lenguaje, a medida que se repite, se implica y se confirma a sí mismo, nos proporcione de manera irrecusable la traza y los contornos de un universo de sentido" [ «le langage ne dit jamais rien, il invente une gamme de gestes qui présentent entre eux des différences assez claires pour que la conduite du langage, à mesure quelle se répète, se recoupe et se confirme elle-même, nous fournisse de manière irrécusable, l'allure et les contours d'un univers de sens »] (pp. 46-47). 
Todo esto nos invita a pensar que una posible solución al problema de la expresión del mundo sensible debe ser buscada en un particular uso del lenguaje literario 4 . Para explorar dicha solución delimitaremos el concepto de gran prosa y, luego, elucidaremos la capacidad propia del lenguaje literario para expresar la verdad. Hacia el final del artículo, esbozaremos algunas consideraciones finales y perspectivas sobre la relevancia de la expresión literaria en el quehacer filosófico y la posibilidad de elaborar una filosofía de lo sensible comprendida en los términos de la literatura.

\section{El concepto merleaupontiano de gran prosa}

EL SER-EN-LA-VERDAD no es distinto del ser en el mundo. La mentada verdad a la que Merleau-Ponty se refiere no es otra cosa que la expresión de la estructuración presente de un determinado ámbito de nuestra realidad. El lenguaje literario, que se manifiesta plenamente en el trabajo del escritor, tiene como función primordial conquistar aquellas regiones foráneas, innombradas, esto es, estructurar en la palabra el sentido naciente del mundo en que nos encontramos (Merleau-Ponty 2013, p. 61).

La expresión de la verdad está íntimamente ligada al lenguaje instituyente característico de la tarea del escritor. En el Primer Coloquio Internacional de Fenomenología Merleau-Ponty (1960) sostenía que

decir que hay una verdad es decir que, cuando mi reasunción encuentra el proyecto antiguo o extranjero y cuando la expresión lograda libera aquello que estaba cautivo en el ser desde siempre, en el espesor del tiempo personal e interpersonal se establece una comunicación interior por la cual nuestro

4 F. Robert (2008) advierte que: "Es significativo que aquel curso [el del mundo de sensible y el mundo de la expresión] vaya acompañado de una meditación sobre el lenguaje literario, es decir, sobre la escritura, y también [...] sobre la escritura no solamente como forma singular de expresión, y de expresión de lo sensible en particular, sino además como lugar posible de emergencia de la verdad, pasaje a la idealidad o a la universalidad de sentido" [ «Il est significatif que ce cours s'accompagne alors d'une méditation sur le langage littéraire, c'est-à-dire aussi sur l'écriture, c'est-à-dire aussi [...] sur l'écriture en tant non seulement que forme singulière d'expression, et d'expression du sensible en particulier, mais en tant également que lieu possible d'émergence de la vérité, passage à lidéalité ou à l'universalité du sens. »] (p. 149). 
presente se convierte en la verdad de todos los demás acontecimientos cognoscentes [...]. En ese momento se ha fundado alguna cosa en su significación, una experiencia se ha transformado en su sentido, ha devenido verdad. La verdad es otro nombre de la sedimentación, que es a su vez la presencia de todos los presentes en el nuestro (p. 120; énfasis añadido).

Merleau-Ponty considera que la verdad está indefectiblemente ligada a la expresión que la manifiesta. Para comprender el dinamismo de la verdad el fenomenólogo apela al concepto de institución (Stiftung), que le permite explicar cómo la verdad supone la fundación de una significación que, posteriormente, puede ser retomada en pos de nuevas instituciones de sentido. Esto puede advertirse con facilidad en el uso que hace el escritor del lenguaje instituido, quien, mediante una "desviación" de las significaciones de su uso instituido, logra segregar-instituir- una nueva significación ${ }^{5}$.

En La prosa del mundo, Merleau-Ponty intentaba ofrecer una teoría de la verdad. Allí, su objetivo era sostener que la comunicación en la literatura no es una simple apelación del escritor a significaciones que formarían parte de un $a$ priori del espíritu humano, sino que ellas son quienes suscitan en él su aparición por seducción o por una suerte de acción oblicua. Mientras trabajaba en ese manuscrito, que sería póstumamente publicado, Merleau-Ponty sugería en un texto preparado para su candidatura en el Collège de France el concepto de gran prosa:

Toda gran prosa es también una recreación del instrumento significante, en adelante manejado según una sintaxis nueva. Lo prosaico se limita a tocar por signos convenidos significaciones ya instaladas en la cultura. La gran prosa es el arte de captar un sentido que no había jamás sido objetivado hasta aquí y de hacerlo accesible a todos aquellos que hablan la misma lengua (MerleauPonty, 2000, p. 45)'.

5 Para ahondar en el problema de la verdad en la obra de Merleau-Ponty y, más precisamente, en el de la institución de sentido que posibilita el lenguaje, sea el de la literatura como el de la matemática, véase "Merleau-Ponty y el problema de la verdad: la historia del pequeño Gauss y la suma de los ángulos del triángulo” (Buceta, 2018b).

6 «Toute grande prose est aussi une recréation de l'instrument signifiant, désormais manié selon une syntaxe neuve. Le prosaïque se borne à toucher par des signes convenus des significations déjà installées 
Con el concepto de gran prosa el autor designa aquella tarea propia del lenguaje creativo mediante el cual es posible referir el mundo indirectamente. Toda gran prosa logra recrear el lenguaje de tal modo que lo hace decir algo que aún no se había expresado. Se vale del lenguaje instituido, conocido por todos, para descentrarlo de su significado usual y reenviarnos hacia una nueva significación. La gran prosa es el arte de captar un sentido que no había sido jamás objetivado, esto es, el sentido naciente del mundo que se da en el encuentro de la carne del cuerpo con la carne del mundo. En ese entrecruzamiento o superposición (empiétement) surge el sentido de lo percibido, y la tarea del escritor es la de llevar a la expresión tal experiencia.

La noción de carne (chair) debe comprenderse dentro del marco general de la reformulación merleaupontiana de la ontología que se da en su inconcluso libro Lo visible y lo invisible (1964). Allí Merleau-Ponty proponía una nueva ontología, estructurada a partir de los conceptos de carne, reversibilidad y quiasmo, que intentaba dar cuenta del surgimiento del sentido a partir del encuentro del vidente con lo visible. Tal encuentro -el del cuerpo con el mundo- debía comprenderse como el de dos espejos enfrentados que se reflejan uno a otro infinitamente y que hacen una pareja más real que cada uno de ellos. En esa "visibilidad", que se instaura en el mutuo reflejarse de lo visible y el vidente, se da el enroscamiento (enroulement) de la carne del mundo con la carne del cuerpo y es posible la institución del sentido mediante la creación de significaciones, de palabras, que recojan el sentido que las ha visto nacer. Para expresar dicha institución, Merleau-Ponty (1964) explica que

el enroscamiento en él [sujeto viviente] de lo visible y de lo vivido sobre el lenguaje, del lenguaje sobre lo visible y lo vivido, los intercambios entre las articulaciones de su paisaje mudo y las de su palabra, en fin, ese lenguaje operante que no necesita ser traducido en significaciones y pensamientos, ese lenguaje-cosa que vale como arma, como acción, como ofensa y como seducción, porque hace aflorar todas las relaciones profundas de lo vivido donde se ha formado, y que es el de la vida y de la acción, pero también el de

dans la culture. La grande prose est l'art de capter un sens qui n'avait jamais été objectivé jusque-là et de le rendre accessible à tous ceux qui parlent la même langue. » 
la literatura y la poesía, ese logos es, pues, un tema absolutamente universal, es el tema de la filosofía. Ella misma es el lenguaje, descansa en el lenguaje; por eso no la descalifica para hablar del lenguaje ni para hablar del pre-lenguaje y del mundo mudo que los dobla: por el contrario, ella es lenguaje operante, ese lenguaje-allí que no puede saberse, sino desde adentro, por la práctica, que está abierto a las cosas, llamado por las voces del silencio, y continúa un ensayo de articulación que es el Ser de todo ser (pp. 165-166). ${ }^{7}$

Este lenguaje operante que logra recoger el sentido del mundo mudo de la percepción es el propio de la gran prosa y es aquel que loaba Merleau-Ponty (1964) en la escritura proustiana cuando afirmaba que "nadie ha superado a Proust en la instauración de las relaciones entre lo visible y lo invisible, en la descripción de una idea que no es lo contrario de lo sensible, que es su doblez y profundidad" (p. 193) ${ }^{8}$. Merleau-Ponty encontraba en la literatura proustiana la capacidad de dar cuerpo a las verdades que emergen en el encuentro con el mundo. En las páginas de una gran prosa se realiza la exploración de un invisible que no puede ser desprendido de las apariencias sensibles a las que está ligado, esto es, que no puede ser comprendido sin pasar por la intuición que ha abierto ese abanico de ideas de ella surgidas?

$7 \quad$ «lénroulement en lui du visible et du vécu sur le langage, du langage sur le visible et le vécu, les échanges entre les articulations de son paysage muet et celles de sa parole, enfin ce langage opérant qui n'a pas besoin d'être traduit en significations et en pensées, ce langage-chose qui vaut comme arme, comme action, comme offense et comme séduction, parce qu'il fait affleurer tous les rapports profonds du vécu où il s'est formé, et qui est celui de la vie et de l'action, mais aussi celui de la littérature et de la poésie, alors ce logos est un thème absolument universel, il est le thème de la philosophie. Elle-même est langage, repose sur le langage; mais cela ne la disqualifie ni pour parler du langage, ni pour parler du pré-langage et du monde muet qui les double: au contraire, elle est langage opérant, ce langage-là qui ne peut se savoir que du dedans, par la pratique, est ouvert sur les choses, appelépar les voix du silence, et continue un essai d'articulation qui est l'Être de tout être. »

8 «Personne n'a été plus loin que Proust dans la fixation des rapports du visible et de l'invisible, dans la description d'une idée qui n'est pas le contraire du sensible, qui en est la doublure et la profondeur. »

9 Cuando hablamos de la intuición que ha abierto el abanico de ideas de ella surgidas, nos referimos al sentido que Merleau-Ponty otorga a esta noción en Lo visible y lo invisible (1964), al afirmar que "con la primera visión, el primer contacto, el primer placer, hay iniciación, es decir, no posición de un contenido, sino apertura de una dimensión que ya no podrá cerrarse, establecimiento de un nivel al que, de ahora en más, será referida toda nueva experiencia” [ Avec la première vision, le 
En la obra de arte, y sobre todo en el caso particular de la novela, el lenguaje ostenta su capacidad de introducirnos a perspectivas extrañas, distintas de las nuestras. En Signos, Merleau-Ponty afirmaba que

lo que no es reemplazable en la obra de arte, lo que hace de ella mucho más que un medio de placer: un órgano del espíritu, cuyo análogo se encuentra en todo pensamiento filosófico o político, si es productivo, es que contiene, más que ideas, matrices de ideas, que nos proporciona emblemas de los que nosotros no hemos nunca terminado de desarrollar el sentido, que, justamente puesto que se instala y nos instala en un mundo del que nosotros no tenemos la clave, nos enseña a ver y finalmente nos da qué pensar como ninguna obra analítica puede hacerlo, porque el análisis no encuentra en el objeto más que lo que hemos puesto en él (Merleau-Ponty 1960, pp. 96-97) ${ }^{10}$.

La obra de arte es considerada por el autor un órgano del espíritu porque es una herramienta con la que contamos para expresar nuestro ser en el mundo. Es un órgano que tiene por función decir nuestro ser en el mundo, que no es otra cosa que la verdad ${ }^{11}$. A diferencia de la producción analítica -que encuentra en el mundo todo lo que ha puesto de antemano-, la obra de arte implica la exploración de la experiencia que, para expresarse, necesita del gesto creativo que

premier contact, le premier plaisir, il y a initiation, c'est-à-dire, non pas position d'un contenu, mais ouverture d'une dimension qui ne pourra plus être refermée, établissement d'un niveau par rapport auquel désormais toute autre expérience sera repérée. »] (p. 196). La intuición es esa primera visión, ese primer contacto, que abre un abanico de ideas que, posteriormente, constituirán ese nivel, ese invisible de este mundo que lo habita, lo sostiene y lo hace visible.

10 « Ce qui n'est pas remplaçable dans l'oeuvre d'art, ce qui fait d'elle beaucoup plus qu'un moyen de plaisir: un organe de l'esprit, dont l'analogue se retrouve en toute pensée philosophique ou politique si elle est productive, c'est qu'elle contient, mieux que des idées, des matrices d'idées, qu'elle [97] nous fournit d'emblèmes dont nous n'avons jamais fini de développer le sens, que, justement parce qu'elle s'installe et nous installe dans un monde dont nous n'avons pas la clef, elle nous apprend à voir et finalement nous donne à penser comme aucun ouvrage analytique ne peut le faire, parce que l'analyse ne trouve dans l'objet que ce que nous y avons mis. »

11 Hacia el final del capítulo sobre "el cogito" de la Fenomenología de la percepción, Merleau-Ponty (1945) afirmaba que "no tenemos la experiencia de un verdadero eterno y de una participación en lo Uno, sino de actos concretos de reasunción, por los cuales, en el azar del tiempo, establecemos relaciones con nosotros mismos y con el otro, en una palabra, la experiencia de una participación en el mundo, el 'ser-en-la-verdad' no es distinto del ser en el mundo” (p. 452). 
logre sublimar su sentido. En la novela el mundo resuena, logra rehacer el sentido del mundo percibido y estructurarlo en el lenguaje proferido. Cuando el escritor utiliza las palabras para llenar ese vacío de lo que se quiere decir, apela, justamente, a aquellas palabras que "transportan a quien habla y a quien las entiende a un universo común, arrastrándolas a un significado nuevo por una potencia de designación que excede su definición recibida" (Merleau-Ponty, 1969, pp. 122-123) ${ }^{12}$. El escritor, por medio de la obra, nos revela y se revela un sentido salvaje que permanecía oculto y que ha sido posible desentrañar por medio de la creación $\operatorname{artística~}^{13}$.

La novela es el género al que se refiere Merleau-Ponty al delimitar el concepto de gran prosa. No es casual que los autores a los que él hace referencia como Proust, Stendhal, Simon, etc., sean ilustres representantes de tal género. En la prosa de estos autores $-y$ de cualquier otro que logre recrear el instrumento significante para hacerlo decir aquello que permanecía mudo- asistimos a un fenómeno maravilloso: el escritor es él mismo como un nuevo idioma que se construye y se inventa medios de expresión para elaborar una gran prosa, el arte de captar un sentido que nunca hasta el momento había sido objetivado y lo hace accesible a todos aquellos que hablan la misma lengua. En relación al trabajo del escritor, Merleau-Ponty escribía sobre Claude Simon que, "sentir, vivir, la vida sensorial es como un tesoro, pero que no vale aún nada antes de que haya sido trabajado. El trabajo no consiste únicamente, por otra parte, en 'convertir en palabras' lo vivido: se trata de hacer hablar lo que se siente" (Merleau-Ponty, 2000, p. 313).

$\mathrm{Al}$ sumergirnos en la lectura de una gran prosa, nos dejamos conducir hacia donde los signos nos indican y rehacemos con el escritor, a cada palabra, la pareja

12 «transportent celui qui parle et celui qui les entend dans un univers commun, mais ils ne le font qu'en nous entraînant avec eux vers une signification nouvelle, par une puissance de désignation qui dépasse leur définition ou leur signification reçue. »

13 En el curso sobre El problema de la palabra, Merleau-Ponty (1953-1954) se propone, entre otras cosas, estudiar en el escritor: "la función conquistadora de la palabra, su capacidad de expresión (Ombredane) como recreación del contexto por el texto. [Ya que] es indudablemente en la literatura que la palabra creadora se encuentra en su plenitud" (p. 93) [ « La fonction conquérante de la parole, sa capacité d'expression (Ombredane) comme recréation du contexte par le texte. C'est évidemment dans la littérature que la parole créatrice est à son comble. »] 
del ciego y el paralítico. Con esta metáfora Merleau-Ponty (1969) nos invita a pensar la relación del lector con el texto y con el autor:

Pero esta es precisamente la virtud del lenguaje: nos arroja sobre lo que significa; se disimula a nuestros ojos en su misma operación; su triunfo está en borrarse y darnos acceso, más allá de las palabras, al pensamiento mismo del autor, de tal suerte que retrospectivamente creamos haber estado conversando con él sin palabras, de espíritu a espíritu. Las palabras una vez enfriadas vuelven a caer sobre la página reducida a simples signos, y precisamente porque nos han proyectado muy lejos de sí, nos parece increíble que tantos pensamientos nos hayan podido venir de ellas. Sin embargo, son los vocablos los que nos han estado hablando, durante la lectura, cuando, sostenidos por el movimiento de nuestra mirada y de nuestro deseo, pero a la vez sosteniéndole y no dándole tregua, rehacían con nosotros la pareja del ciego y el paralítico -cuando eran gracias a nosotros, y nosotros éramos gracias a ellos, palabra más que lenguaje, la voz y su eco al mismo tiempo (pp. 16-17) $)^{14}$.

Merleau-Ponty desliza sutilmente la metáfora del ciego y el paralítico para ilustrar la relación entre lector y autor. La explicación es simple, el paralítico -el autor- va guiando al ciego -el lector- hacia esa idea que quiere revelar y que se encuentra incrustada en el mundo sensible al que ambos pertenecen. El escritor confecciona una gran prosa en la que, mediante el arreglo de determinadas palabras, logra encarnar las ideas que se hallan inseparablemente unidas a la experiencia en que se manifiestan y que migran de la carne del mundo a la carne menos pesada del lenguaje. El lector irá empujando la silla del paralítico hacia donde este le indique mediante signos y así ambos descubrirán ese pensamiento que solo puede hacerse en el lenguaje, irán transitando el camino hacia ese lugar

14 «Mais cela même est la vertu du langage : c'est lui qui nous jette à ce qu'il signifie; il se dissimule à nos yeux par son opération même; son triomphe est de s'effacer et de nous donner accès, par-delà les mots, à la pensée même de l'auteur, de telle sorte qu'après coup nous croyons nous être entretenus avec lui sans paroles, d'esprit à esprit. Les mots une fois refroidis retombent sur la page à titre de simples signes, et justement parce qu'ils nous ont projetés bien loin d'eux, il nous semble incroyable que tant de pensées nous soient venues d'eux. C'est pourtant eux qui nous ont parlé, à la lecture, quand, soutenus par le mouvement de notre regard et de notre désir, mais aussi le soutenant, le relançant sans défaillance, ils refaisaient avec nous le couple de l'aveugle et du paralytique, - quand ils étaient grâce à nous, et nous étions grâce à eux parole plutôt que langage, et d'un seul coup la voix et son écho. » 
desconocido. El lenguaje conquistador de la gran prosa confeccionada por el paralítico será el conjunto de signos que llevarán al ciego hacia esa región del mundo innombrada, a la institución de una verdad del mundo vivido que se realiza en el momento de la expresión literaria. Dicha verdad trasparece en la expresión lograda, allí cuando el lenguaje consigue arrojarnos sobre lo que significa, cuando triunfa al darnos acceso al mundo al que se refiere.

Merleau-Ponty (2003) afirma que, "las palabras del escritor descentran la significación usual y a través de usos convergentes indican nuevos sentidos que son desviaciones" (p. 90) ${ }^{15}$. El escritor utiliza su lenguaje, y la significación de las palabras que él emplea es lo que ellas arrastran consigo de las configuraciones de su paisaje.

Así como el pintor y el músico se sirven de objetos, colores y sonidos para manifestar las relaciones de los elementos del mundo dentro de la unidad de una vida -por ejemplo, las correspondencias metafóricas de un paisaje marino-, el escritor, tomando el lenguaje de todos, se sirve de él para traducir la participación prelógica de los paisajes, de las moradas, de los lugares, de los gestos, de los hombres entre ellos y con nosotros (Merleau-Ponty, 1968, pp. 39-40).

Una novela es una gran construcción en la que los acontecimientos relatados son puestos en relación y su estructuración instaura un sentido que permanecía latente y ahora se hace manifiesto. El escritor se vale del lenguaje que tenemos en común para manifestar los paisajes ${ }^{16}$ de la experiencia, llevándolos a la expresión mediante una utilización creativa de los signos; "es él mismo como un nuevo idioma que se construye, se inventa medios de expresión y se diversifica según su propio sentido" (Merleau-Ponty, 2000, p. 45). Su trabajo supone la elaboración

15 « les mots de l'écrivain décentrent la signification usuelle et par usages convergents indiquent nouveaux sens qui sont écarts. »

16 Respecto a la noción de "paisaje", la comprendemos a la manera de Collot (1997): "reúne la imagen del mundo con la actividad de una conciencia encarnada; el paisaje es la patria tal como aparece a la mirada de un sujeto cuyo punto de vista lo organiza como un conjunto con forma y sentido" [« il réunit aussi l'image du monde à l'activité d'une conscience incarnée : le paysage, c'est le pays tel qu'il apparaît au regard d'un sujet dont le point de vue l'organise comme un ensemble ayant forme et sens. »] (p. 26). Para ahondar en este término en la filosofía merleaupontiana y, en particular, en la tarea del escritor, puede verse "L'œuvre comme paysage d'une expérience. Merleau-Ponty et la critique thématique” (Collot, 1997, pp. 23-37). 
de ese paisaje que será el fondo sobre el cual determinados gestos y palabras cobrarán un sentido único, se destacarán. El lector es introducido por el lenguaje conquistador del escritor en perspectivas extrañas, novedosas. A medida que avanza en la lectura es dirigido hacia donde los signos le indican, descubre una idea que solo puede ser expresada por medio de los giros y desviaciones elaborados por la obra y que es inseparable de su expresión ${ }^{17}$. El escritor

nos transporta del mundo ya dicho a otra cosa. Y de la misma manera que nuestro cuerpo no nos guía entre las cosas, sino a condición de que cesemos de analizar para hacer uso de él, el lenguaje no es literario, es decir productivo, sino a condición de que cesemos de pedirle a cada momento justificaciones para seguirle a donde él va, de que dejemos a las palabras y a todos los medios de expresión del libro rodearse de esa aureola de significado que deben a su disposición singular (Merleau-Ponty, 1960, pp. 97) ${ }^{18}$.

17 Las ideas sensibles son aquellas que no pueden ser separadas del tejido de la experiencia en que nos son dadas. Merleau-Ponty (1964) afirma que "la literatura, la música, las pasiones, tanto como la experiencia del mundo visible son, no menos que la ciencia de Lavoisier y de Ampère, la exploración de un invisible y, como ella, develamiento de un universo de ideas. Simplemente, ese invisible, esas ideas, no se dejan, como las de aquellos, desprender de las apariencias sensibles, ni erigir en positividad secundaria" [ « La littérature, la musique, les passions, mais aussi l'expérience du monde visible, sont non moins que la science de Lavoisier et d'Ampère l'exploration d'un invisible et, aussi bien qu'elle, dévoilement d'un univers d'idées. Simplement, cet invisible-là, ces idées-là, ne se laissent pas comme les leurs détacher des apparences sensibles, et ériger en seconde positivité. »] (pp. 193-194). Las ideas son inescindibles de su aparecer sensible y por ello son llamadas por el filósofo francés ideas sensibles, porque es justamente a través de lo sensible, por medio de lo sensible, que esas ideas aparecen. No es fuera de la experiencia, detrás de la contingencia, sino en y por ella que aprendemos las ideas que se encuentran en su entramado. Y estas no pueden ser desprendidas de él, sino, solamente, advertidas y expresadas, sin ser separadas del medio en que existen y sin el que no podrían ser comprendidas. Estas ideas funcionan como niveles que se establecen al contacto con lo sensible, abriendo una dimensión que jamás podrá cerrarse y en relación con la cual una serie de acontecimientos será comprendida. Para una elucidación de este concepto central del último período de la filosofía merleaupontiana, véase Buceta, 2018a, pp. 123-148; Farrés Famadas, 2011, pp. 79-93; y Carbone, 2008, pp. 53-80; 2012, pp. 99-11; 2015, pp. 59-82.

18 « nous transporte du monde déjà dit à autre chose. Et comme notre corps ne nous guide parmi les choses qu'à condition que nous cessions de l'analyser pour user de lui, le langage n'est littéraire, c'està-dire productif, qu'à condition que nous cessions de lui demander à chaque instant des justifications pour le suivre où il va, que nous laissions les mots et tous les moyens d'expression du livre s'envelopper de cette auréole de signification qu'ils doivent à leur arrangement singulier. » 
Para que el lenguaje pueda ser considerado literario es preciso que nos abandonemos a sus directrices y, de este modo, se irá deslindando un universo de sentido que trasparece en la lectura de la gran prosa y es inseparable de las construcciones literarias en las que se nos da. El autor dispone las significaciones de un determinado modo, logrando tejer, minuciosamente, una gran red en que se entremezclan personajes con determinadas características, lugares, situaciones, pensamientos; va construyendo todo un universo que se prepara para dar a luz a aquel sentido que se quiere poner de manifiesto y que precisa de todo ese trabajo previo, de todo ese mundo sin el que no podría ser comprendido lo que se quiere decir.

El lenguaje literario expresa de este modo, no solo por lo que se dice, sino también por los silencios, por lo que no se dice. El lenguaje indirecto, propio de la literatura, se vale de giros, formas y ambigüedades que expresan rodeando la significación, sin querer abordarla directamente. Merleau-Ponty se refería a esto cuando explicaba que "hablar poéticamente del mundo es casi callarse" (2002, p. 59 ${ }^{19}$. El silencio no es lo contrario del lenguaje, sino que lo conforma y lo asiste en la tarea de conducirnos, de modo indirecto, lateral, hacia la expresión. El lenguaje literario, hablante -que más adelante explicitaremos- nos habla del mundo como susurrándonos, expresa con sus palabras indirectas y con sus silencios elocuentes.

La novela se nos aparece como un todo que se va descubriendo palabra a palabra, frase a frase, y en la que, lentamente, si nos dejamos llevar por él, el escritor va edificando ese universo en que cada personaje es alguien, cada sitio un lugar y el sentido aparece porque las palabras no se lanzan al vacío, sino que resuenan sobre la gran sinfonía de la novela. El escritor se acomoda en nuestro lenguaje común, en el que estamos iniciados por el simple hecho de pertenecer al mundo y tener un cuerpo y, desde ese lugar, recrea el instrumento significante, realiza una torsión secreta para dar a luz a un sentido que permanecía oculto.

El sentido de un libro está dado, primeramente, no tanto por las ideas, sino por una variación sistemática e insólita de los modos del lenguaje y del relato, o de las formas literarias existentes. Ese acento, esa modulación particular de la palabra, si la expresión es lograda, es asimilada poco a poco por el lector 
y le hace accesible un pensamiento al que era alguna vez indiferente o más rebelde en principio (Merleau-Ponty, 2000, p. 44) ${ }^{20}$.

\section{La literatura y la expresión de la verdad}

Merleau-Ponty, a lo largo de su obra, fue otorgando cada vez mayor importancia a la consideración de la expresión literaria. Esto puede constatarse, primero, por el ineludible capítulo sobre "El cuerpo como expresión y la palabra" de la Fenomenología de la percepción, donde ya abordaba el fenómeno del lenguaje estableciendo una distinción hacia dentro del mismo entre palabra hablada (parole parlée) y palabra hablante (parole parlante) (Merleau-Ponty, 1945, p. 229). Luego, puede advertirse una preocupación creciente en La prosa del mundo (1969, pp. 66-160) y Signos (1960, pp. 49-122), textos en que se ocupa del problema del "lenguaje indirecto" y su poder de instituir nuevas significaciones, en alusión al lenguaje propio de la literatura. Estos escritos suponen una reflexión en torno a la posibilidad de considerar la literatura como ámbito privilegiado para develar la verdad -problema que se torna central en "El algoritmo y el misterio del lenguaje” (Merleau-Ponty, 1960, pp. 161-181)-21.

20 «Le sens d'un livre est premièrement donné, non tant par les idées, que par une variation systématique et insolite des modes du langage et du récit, ou des formes littéraires existantes. Cet accent, cette modulation particulière de la parole, si l'expression est réussie, est assimilée peu à peu par le lecteur et lui rend accessible une pensée à laquelle il était quelquefois indifférent ou même rebelle d'abord.»

21 Para ahondar en la deriva de los estudios merleaupontianos en torno a la literatura puede consultarse Simon y Castin (Eds.), 1997, quienes han compilado las actas correspondientes al coloquio Merleau-Ponty et le littéraire. En estos trabajos, en los que se busca examinar la mutua influencia entre la reflexión merleaupontiana sobre la creación literaria y el avance de lo literario sobre su filosofía, se advierte que existe en Merleau-Ponty una fascinación determinante por los escritores en los que se halla "una palabra literaria que incluye la efervescencia sensible" [ « une parole littéraire qui inclut l'effervescence sensible »] (p. 14). Son destacables las contribuciones de Collot (pp. 23-38), "La obra como paisaje de una experiencia. Merleau-Ponty y la crítica temática", en la que el autor busca comprender la articulación de la escritura, la lectura y la experiencia sensible y advierte en las bases sentadas por la fenomenología merleaupontiana la posibilidad de pensar "esa inscripción de lo sensible en el texto" [《cette inscription du sensible dans le texte »] (p. 24); y la de Neefs (pp. 117-131), "El estilo es visión (Merleau-Ponty y Claude Simon)", en que el autor aborda las incipientes lecturas merleaupontianas de la obra de Simon analizando las "cinco notas sobre 
Sin embargo, existe un curso hasta hace poco inédito, dictado por el filósofo francés en el Collège de France, durante el año académico 1953-1954, en que el tema de la capacidad de la literatura para expresar la verdad es principal. Merleau-Ponty titula ese curso El problema de la palabra (Le problème de la parole). En los Resúmenes de curso publicados afirmaba que "el curso buscaba ilustrar y extender esta noción saussureana de la palabra como función positiva y conquistadora" (Merleau-Ponty, 1968, p. 34). El resumen es breve e introductorio, pero aparecen allí algunos indicios sobre los temas centrales de los que se ocupará en sus clases, por ejemplo, la figura del escritor quien, según Merleau-Ponty (1968), "trata de producir un sistema de signos que restituya, gracias a su ordenamiento interno, el paisaje de una experiencia” (p. 40). El problema de la palabra es el intento de desentrañar el fenómeno del lenguaje literario para demostrar su función conquistadora de verdades: "la literatura tiene por función conquistar una verdad” (Merleau-Ponty, 1953-1954, p. 119v) 22. Esta verdad que se manifiesta en el leguaje conquistador de una gran prosa se encuentra siempre en las entrañas del mundo vivido. Por lo tanto, el objeto de la literatura será revelar dicho mundo, ya que ella se perfila como el modo universal de contacto mudo e individual con los otros y las cosas (Merleau-Ponty, 1953-1954, p. 119).

En el curso en cuestión, la figura de Marcel Proust es principal; se analizan allí diversos pasajes de la Recherche para caracterizar la acción conquistadora de la literatura. Al igual que Proust, Merleau-Ponty descubría en el lenguaje literario una primordial vocación por la verdad y su expresión, y veía en la gran prosa la capacidad de transformación del mundo vivido en lenguaje. Para Merleau-Ponty, "el objeto de la literatura es transformar en 'lenguaje universal' el mundo vivido. Se trata de vocación de la verdad” (1953-1954, p. 112v). Esta misma motivación es la que advierte el Narrador de la Recherche cuando descubre su vocación

Claude Simon" escritas por el filósofo, y deduce de estas la convicción de que "la literatura ofrece esa irremplazable pertenencia a las propiedades del mundo, ella es una experiencia formulada de la aún no formulada, a la vez origen y receptáculo de una experiencia fundamental: 'ella se dirige a (viene de) esa región por debajo de las ideas" [ « la littérature offre cette irremplaçable appartenance aux propriétés du monde, elle est une expérience formulée de l'encore-informulé, à la fois origine et réceptacle d'une expérience fondamentale: "elle s'adresse à (vient de) cette région au-dessous des idées". »] (p. 120). 
literaria y, a su vez, a la literatura como la verdadera vida, como ámbito en que el mundo puede ser recobrado, su verdadera esencia ser expresada ${ }^{23}$. María del Carmen López Sáenz (2008) señala este objetivo común de ambos autores:

Proust [...] entiende la novela como una construcción, como un todo cuyas ideas vertebrales no han de exponerse exhaustivamente, sino hacerse notar a través del estilo, que las individualiza. Merleau-Ponty persiguió objetivos similares por la vía de la filosofía; en el fondo, aspiraban a la misma verdad -cada uno por su senda-, a la búsqueda de sentido en el sinsentido, al pensamiento y a la expresión de lo vivido [...]. Sus verdades indagadas no eran la Verdad, ni la adecuación de las ideas espirituales con las cosas materiales; eran verdades invisibles, pero reales, porque acercaban al otro lado de lo visible, a la profundidad de este, a las ideas sensibles a las que nos referimos (p. 317).

En el caso particular de Proust, podemos hallar el pasaje a la idea por medio del acto de la expresión creativa que se desenvuelve en su obra. "Si Proust es, para Merleau-Ponty, filósofo, es porque hace de la literatura el modo indirecto de conquistar esa verdad que está por hacer" (López Sáenz, 2008, p. 309). Nos encontramos comprometidos en un mundo en el que nuestro cuerpo busca expresar su situación. La gran prosa aparece, entonces, como una de las herramientas principales para esta tarea, porque "literatura es conversión de esa coexistencia, elaboración a partir de ella de un sistema de lenguaje" (Merleau-Ponty, 19531954, p. 126) $)^{24}$.

La clave para esta reconversión del mundo vivido en la expresión es la palabra (parole). La palabra es parte del mundo sensible, pero se prolonga en lo inteligible. Ella es relación con el Ser a través de un ser. El lenguaje hablante, conformado por palabras conquistadoras, es la dimensión del sujeto en la que se da la sublimación de la experiencia muda del encuentro con el mundo, el quiasmo en que el mundo mudo se entrecruza y se confunde con el mundo de la expresión.

23 Esta "vocación de verdad" que señala Merleau-Ponty en la literatura es lo que encontramos principalmente en $A$ la busca del tiempo perdido, que, como algunos estudiosos de la obra proustiana afirman, debería titularse $A$ la busca de la verdad -véase por ejemplo, Descombes, 1987, pp. 12-13; Deleuze, 1964, p. 23; y Compagnon, 1987, p.9-.

24 «la littérature est conversion de cette coexistence, élaboration à partir d'elle d'un système de langage. » 
La palabra es un ser del mundo visible y, al mismo tiempo, abre a lo invisible. Este concepto es fundamental en la propuesta merleaupontiana para explicar la reconversión del silencio en lenguaje, para dar cuenta de la sublimación de la experiencia del mundo de la percepción en el mundo de la expresión ${ }^{25}$. La palabra será en la producción literaria la que posibilite la manifestación de la verdad, esto es, la vehiculización del ser-en-el-mundo del cuerpo propio hacia la expresión. Según Merleau-Ponty (1953-1954),

la palabra tendrá por función tomar por tema esa aparición del mundo en la trascendencia, esa estructura perspectiva-realidad, esa presencia en la perspectiva justamente de la realidad, [...] esa presentación lateral, por perfil, que es la garantía de totalidad situada más allá de ella -todo eso reposando sobre nuestra encarnación en un punto de vista (sin el que no habría perspectiva alguna), sobre nuestra espacialidad- [...] (p. 95v $)^{26}$.

La palabra realiza el vínculo entre lo vivido y lo expresado, el quiasmo entre lo visible y lo invisible. La palabra hablante de la literatura articula el silencio sonoro de las cosas del mundo de la percepción para quienes saben escucharlo. Existe entre el cuerpo y las cosas una complicidad, un pacto tácito, una continuidad que solo puede ser dicha de modo indirecto. Merleau-Ponty (1953-1954) entiende que "hay allí una continuidad. Los poderes de la literatura no son distintos que los de la palabra: una relación preobjetiva al mundo y al otro" (p. 101) ${ }^{27}$.

Los actos de expresión dejan huellas que pueden, posteriormente, ser reutilizadas por un espíritu capaz de llevar a cabo una recuperación de lo que en ellas se encuentra cautivo. Lo sedimentado en la palabra es el sentido del mudo que se encuentra allí contraído y que es posible recobrar en favor de nuevas

25 Para ahondar en el problema del pasaje del mundo sensible al mundo de la expresión puede verse nuestro trabajo, titulado "Analogía y sublimación: una respuesta al problema del pasaje del silencio a la palabra en la filosofía de Merleau-Ponty” (Buceta, 2017, pp. 333-360).

26 « la parole aura pour fonction de prendre pour theme cette apparition du monde dans la transcendance, cette structure perspective-réalité [...] cette présentation latérale, par profil, qui est la garantie de totalité située au-delà d'elle - tout cela reposant sur notre incarnation dans un point de vue (sans laquelle il n'y aurait pas perspective), donc sur notre spatialité. »

27 «Il y a là une continuité. Les pouvoirs de la littérature ne sont pas d'une autre sorte que ceux de la parole : un rapport préobjectif au monde et à autrui. » 
estructuraciones de sentido, de nuevas expresiones del mundo vivido en la palabra (Merleau-Ponty, 1953-1954, p. 91). En una gran prosa, como la proustiana, asistimos a ese acontecimiento en el que el escritor intenta "conquistar por las palabras el contacto mudo [...], hacer accesible a los otros aquello mismo que es mudo" (p. 94v) ${ }^{28}$. Aquella experiencia fundamental de la trascendencia de las cosas, de un mundo silencioso que busca ser expresado, es la que invita al autor a escribir, a decir la verdad del mundo circundante, tal como se presenta en la percepción. Por ello, en las notas preparatorias Merleau-Ponty explica que "escribir no es hablar a alguien, justamente porque se trata, escribiendo, de hacer existir la verdad" (p. 124) ${ }^{29}$. Lo distintivo de las grandes obras reside en la virtud propia del artista, que logra expresar, comunicar a otros, su particular encarnación en el mundo. El estilo de un escritor queda definido por los medios a través de los cuales transforma en lenguaje su experiencia del mundo: es su modo de deformar el mundo. Según Merleau-Ponty, "el estilo es el ensamble de medios por los cuales nosotros transformamos en lenguaje, es decir nosotros volvemos comunicable la textura de nuestra experiencia” (p. 124) ${ }^{30}$. Cada escritor tiene un particular estilo, es decir, un modo de acercase al mundo, de expresar su experiencia de las cosas, y eso delineará su manera de construir su gran prosa.

El acto de escribir, como el acto de pintar, es el intento de restituir la participación metafórica de las cosas entre ellas, de nosotros a las cosas y a los otros. La palabra literaria se prepara por organización de nuestra vida en tanto que ella es confrontación con las otras vidas, constitución de dimensiones, de analogías, de equivalencias, paisaje cada vez más significativo con sus relieves y vectores (p. 125). ${ }^{31}$

\footnotetext{
28 « conquérir par les mots le contact muet [...] rendre accessible aux autres cela même qui est muet. »

29 «écrire n'est pasparler à quelqu'un, justement parce qu'il s'agit, en écrivant, de faire exister la vérité. »

30 «le style est l'ensemble des moyens par lesquels nous transformons en langage, c'est-à-dire nous rendons communicable la texture de notre expérience. »

31 «L'acte d'écrire, comme l'acte de peindre, est l'essai de rendre la participation métaphorique des choses entre elles, de nous aux choses et aux autres - La parole littéraire se prépare par organisation de notre vie en tant qu'elle est confrontation avec les autres vies, constitution de dimensions, d'analogies, d'équivalences, paysage de plus en plus significatif avec ses reliefs, ses vecteurs. »
} 
El escritor construye un sistema de lenguaje que pueda sostener las dimensiones del mundo trascendente de las cosas. La obra del literato es la expresión que, por medio de giros, analogías y el establecimiento de equivalencias, logra restituir el paisaje de la experiencia con sus relieves, desviaciones y relaciones. La expresión es traducción del libro interior de la experiencia en el que descubrimos que las cosas llaman a la palabra, su interior es palabra (Merleau-Ponty, 19531954, p. 96v). Cuando la expresión es feliz, lo que se manifiesta es el sentido naciente del mundo de la percepción. Este sentido se cristaliza en una significación que se instituye y que nos permite alcanzar la verdad del mundo vivido. La palabra del escritor "hace existir un universo para el lector, deviene expresión en el sentido de testimonio, expresión en el sentido de creación, y, precisamente por esa razón, porque reconstituye delante de él la situación que traduce, aparece como diciendo un en sí” (p. 102)32.

El escritor buscará entonces sacudir el lenguaje para hacerlo decir más de lo que está dicho, para expresar un excedente. Dado el aparato preexistente del lenguaje -es decir, el lenguaje instituido- el novelista trabajará para reelaborarlo, reordenarlo y, mediante una torsión secreta, disponerlo de tal modo que logre instituir una significación novedosa capaz de expresar aquel silencio hablante del mundo de las cosas.

Solo se nos puede hablar en un lenguaje que ya comprendemos, cada una de las palabras de un texto difícil despierta en nosotros pensamientos que nos pertenecían de antemano, pero estas significaciones se anudan a veces en un pensamiento nuevo que las readapta todas, nos transportamos al centro del libro, damos con la fuente (Merleau-Ponty, 1945, p. 208). ${ }^{33}$

La novedad en el lenguaje aparece como desvío (écart) sobre un nivel implícito, como una diferencia, en la que algo cobra sentido en relación con el todo. El

32 « elle fait exister un univers pour le lecteur, elle devient d'expression au sens de témoignage, expression au sens de création, et, précisément pour cette raison, parce qu'elle reconstitue devant lui la situation qu'elle traduit, elle apparaît comme disant un en soi. »

33 «On ne peut nous parler qu'un langage que nous comprenons déjà, chaque mot d'un texte difficile éveille en nous des pensées qui nous appartenaient auparavant, mais ces significations se nouent parfois en une pensée nouvelle qui les remanie toutes, nous sommes transportés au centre du livre, nous rejoignons la source. 》 
sentido aparece diacríticamente, como una deformación, no totalmente distinta, sino coherente ${ }^{34}$. En las conclusiones del curso sobre el problema de la palabra Merleau-Ponty (1953-1954) explica este punto, afirmando que

hemos visto que la significación (la "idea" en el sentido de Proust) es siempre desvío entre 2 o más significaciones, aparición de un vacío determinado, no posesión intelectual de un contenido y, por consiguiente, su relación a eso que es dicho no es jamás directa, es siempre oblicua [...]. Ella es la palabra creadora, originaria (p. 135) $)^{35}$.

La literatura, la gran prosa, es el ámbito propicio para el desarrollo dicha palabra creadora originaria. El lenguaje conquistador del escritor es aquel que, mediante el arreglo de los signos, logrará expresar aquellas "esencias alógicas, esto es, no comprendidas, inseparables de la impresión que deja la obra” (Merleau-Ponty, 1953-1954, p. 112) ${ }^{36}$. A la busca del tiempo perdido es para Merleau-Ponty la

34 Sobre la consideración de la aparición del sentido como écart, Merleau-Ponty (1968) explica que "el sentido de la cosa percibida, si se la distingue de todas las otras, no está aún aislado de la constelación en que aparece; solo se pronuncia como cierto desvio [écart] respecto del nivel de espacio, de tiempo, de movilidad y, en general, de significación en que estamos establecidos; solo se da como una deformación - pero sistemática- de nuestro universo de experiencia" [ «e sens d'une chose perçue, s'il la distingue de toutes les autres, n'est pas encore isolé de la constellation où elle apparaît, il ne se prononce que comme un certain écart à l'égard du niveau d'espace, de temps, de mobilité et en général de signification où nous sommes établis, il n'est donné que comme une déformation, mais systématique, de notre univers d'expérience »] ( p. 12). Para ahondar en el concepto de desvío (écart) en la filosofía merleaupontiana véase Alloa, 2014, donde se buscará sostener que Merleau-Ponty elabora - a partir de la lectura de Saussure- "una concepción del sentido que ya no es pensada en términos de referencialidad, sino en los términos de una articulación diacrítica inmanente" (p. 162) ["a conception of meaning which is not thought anymore in terms of referentiality, but in terms of immanent diacritical articulation"], en tanto que "el sentido emerge no de lo sustancial, sino de la relación lateral" (p. 163) [“meaning emerges not from substantial, but from lateral relationship”]. Véase también Kearney, 2014, artículo dedicado a sostener que todas las formas de expresión comparten la función diacrítica y que "el sentido se encuentra allí donde la expresión diacrítica cruza la experiencia de la carne" [ « le sens se trouve là où l'expression diacritique croise l'expérience de la chair. $\gg]$ (p. 183).

35 «nous avons vu que la signification (l'idée" au sens de Proust) est toujours écart entre 2 ou plusieurs significations, apparition d'un vide déterminé, non possession intellectuelle d'un contenu, que par suite, son rapport à ce qui est dit n'est jamais direct, toujours oblique [...] Elle est la parole créatrice, originaire. 》

36 « Essences alogiques i.e. non embrassées, inséparables de l'impression que laisse l'œuvre. » 
novela que ostenta más que ninguna otra la tarea distintiva del lenguaje literario, aquella de la expresión de las esencias del mundo, es decir, de las ideas sensibles. En la Recherche asistimos a la presentación lateral, oblicua, de aquel mundo de la experiencia que trasparece a lo largo de su lectura. Proust recobra aquel mundo perdido, aquella esencia extratemporal del mundo, apelando al poder del lenguaje, que conserva el sentido apresado en sus significaciones y que, mediante una reordenación, es capaz de decir aquella verdad tan ansiada.

Una gran prosa contiene y expresa las ideas sensibles que estructuran la carne de la experiencia. Las grandes novelas logran contener y dar expresión a un sentido que se halla disperso en la experiencia, en tanto que recrean el instrumento significante y captan un sentido que jamás había sido objetivado para hacerlo accesible a todos. La literatura puede así ser considerada una actividad conquistadora de la verdad en tanto logra cumplimentar la tarea que Husserl imponía: llevar a la expresión de su propio sentido a la experiencia aún muda, decir la verdad del mundo.

\section{Consideraciones finales: hacia una filosofía} de lo sensible como literatura

En una nota de trabajo de Lo visible y lo invisible, Merleau-Ponty (1964) afirmaba que

lo sensible es $[. .$.$] como la vida, un tesoro siempre lleno de cosas que decir$ para aquel que es filósofo (es decir, escritor) [...]. El fondo del asunto es que, en efecto, lo sensible no ofrece nada que se pueda decir si no se es filósofo o escritor, pero eso no se debe a que sería un en-sí inefable, sino al hecho de que no se sabe decir" (p. 300). ${ }^{37}$

Esta nota de trabajo inicia una discusión -que a causa de su repentina muerte Merleau-Ponty no pudo desarrollar- en torno a cómo pensar las relaciones entre filosofía y literatura, que se centra, principalmente, en el problema de que la

37 «e sensible est [...] comme la vie, trésor toujours plein de choses à dire pour celui qui est philosophe (c'est-à-dire écrivain [...] Le fond des choses est qu'en effet le sensible n'offre rien qu'on puisse dire si l'on n'est pas philosophe ou écrivain, mais que cela ne tient pas à ce qu'il serait un en Soi ineffable, mais à ce qu'on ne sait pas dire. » 
expresión de lo sensible no reside en su posible inefabilidad, sino en los modos de decirlo. La íntima relación que establece Merleau-Ponty entre la tarea del filósofo y el literato al reunirlos bajo una disyunción inclusiva nos impide hablar ya de disciplinas claramente distinguibles, asimétricas y jerarquizadas, tal como lo ha intentado la tradición de pensamiento filosófico (Sabot, 2002, pp. 5-13). Al contrario, exige una reflexión en torno a un posible entrelazamiento en el que será más oportuno hablar de filósofos-escritores o escritores-filósofos y de una tarea que, como explicábamos en la introducción, comparte el mismo género de atención y la misma voluntad de captar el sentido naciente del mundo.

La filosofía de lo sensible podría comprenderse como literatura en tanto que ella está llamada a descubrir el tesoro de lo sensible y decir con el lenguaje correspondiente -lenguaje literario- el en-sí que parece inefable, pero no lo es. La filosofía y la literatura son modos de cumplir con la tarea interminable de llevar la experiencia aún muda a la expresión de su propio sentido. Esto implica que el punto de partida de ambas supone la captación de lo sensible o -siguiendo el texto del propio Merleau-Ponty- el descubrimiento del tesoro siempre lleno de cosas a decir que supone el mundo sensible. Este tesoro debe ser expresado literariamente; por ello, solo "el filósofo (es decir, el escritor)" podrá llevar dicha experiencia a la expresión propia de su sentido. La filosofía de lo sensible ha de ser como la literatura, en tanto pueda contener en sus expresiones el sentido del mundo vivido y debe aprender del trabajo propio del escritor que, como ya explicitamos, "no consiste más que en 'convertir en palabras' lo vivido; se trata de hacer hablar lo que se siente” (Merleau-Ponty, 2000, p. 313)38.

Anteriormente señalamos que Merleau-Ponty (1960) asignaba a la filosofía una tarea específica, al afirmar que la obra de arte "contiene, más que ideas, matrices de ideas, que nos proporciona emblemas de los que nosotros no hemos nunca terminado de desarrollar el sentido [...], nos enseña a ver y finalmente nos da qué pensar como ninguna obra analítica puede hacerlo" (pp. 96-97). El quehacer de la filosofía supone también la exposición, la delimitación, de aquellas matrices de ideas que se dan en la particular expresión artística. El caso paradigmático de esta expresión es para Merleau-Ponty la literatura. En otra nota de $L o$

38 «ne consiste pas seulement, d'ailleurs, à "convertir en mots" le vécu; il s'agit de faire parler ce qui est senti. » 
visible y lo invisible, titulada "Filosofía y literatura", Merleau-Ponty (1964) afirma que "la filosofía, precisamente como 'Ser que habla en nosotros', expresión por sí misma de la experiencia muda, es creación (Gebilde). Creación que es al mismo tiempo reintegración del Ser" (p. 247) ${ }^{39}$. Más adelante, en esa misma nota, agregará que arte y filosofía son formas de contacto con el Ser, en tanto que ambas son creaciones. Creación que busca ser, al mismo tiempo, adecuación con el Ser. Al final de la nota delimita una tarea clara: "hacer análisis de la literatura en este sentido: como inscripción del Ser” (p. 248). Merleau-Ponty considera la literatura en sí misma como un ámbito de inscripción del Ser, como lugar propio de la expresión de una idea, de un motivo invariante, de una verdad.

La relación vislumbradaentre ambas disciplinas no solo es sugerida por Merleau-Ponty al final de su filosofía, sino que también puede encontrarse en sus primeros escritos la consideración de que "la tarea de la literatura y la de la filosofía ya no pueden andar separadas [...]. La expresión filosófica asume las mismas ambigüedades que la expresión literaria, puesto que el mundo está hecho de tal modo que no puede ser expresado más que a través de 'historias' y mostrado como con el dedo" (Merleau-Ponty, 1948, pp. 48-49) ${ }^{40}$.

Una filosofía de lo sensible comprendida en los términos de la literatura es un proyecto que el filósofo augura, pero no concreta. Este pensamiento permanece latente hasta los últimos textos. De hecho, en otra nota de trabajo de noviembre de 1960 -apenas seis meses antes de su muerte- considera una reelaboración de la idea de filosofía en que "lo que ella dice, sus significaciones, no son invisible absoluto: hace ver por palabras. Como toda la literatura. No se instala en el reverso de lo visible: está en ambos lados" (Merleau-Ponty, 1964, p. 313) ${ }^{41}$.

39 «Laphilosophie, précisément comme "Être parlant en nous", expression de l'expérience muette par soi, est création. Création qui est en même temps réintégration de l'Être. »

40 « la tâche de la littérature et celle de la [49] philosophie ne peuvent plus être séparées. Quand il s'agit de faire parler l'expérience du monde et de montrer comment la conscience s'échappe dans le monde, on ne peut plus se flatter de parvenir à une transparence parfaite de l'expression. L'expression philosophique assume les mêmes ambiguïtés que l'expression littéraire, si le monde est fait de telle sorte qu'il ne puisse être exprimé que dans des "bistoires" et comme montré du doigt. »

41 «Ce qu'elle dit, ses significations, ne sont pas de l'invisible absolu : elle fait voir par des mots. Comme toute la littérature. Elle ne s'installe pas dans l'envers du visible : elle est des deux côtés. » 
La incipiente, pero no por ello poco sugerente, relación entre filosofía y literatura ha sido iniciada en la obra merleaupontiana y, tal como acertadamente ha señalado Dimitris Apostolopoulos (2018), "las discusiones académicas sobre la estética de Merleau-Ponty han tendido a concentrarse en su filosofía de la pintura [...]; comparativamente se ha prestado menos atención a la filosofía de la lite-

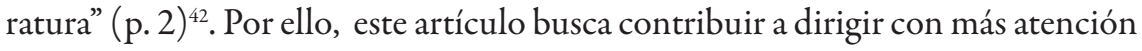
nuestra reflexión hacia la elaboración de una posible filosofía de lo sensible, justamente a partir de la poco estudiada filosofía de la literatura merleaupontiana.

\section{Referencias}

Alloa, E. (2014). The Diacritical Nature of Meaning: Merleau-Ponty with Saussure. Chiasmi International, 15, 167-179. doi: 10.5840/chiasmi20131516

Apostolopoulos D. (2018). The Systematic Import of Merleau-Ponty's Philosophy of Literature. Journal of the British Society for Phenomenology, 49(1), 1-17. doi: 10.1080/00071773.2017.1387683

Buceta M. (2017). Analogía y sublimación: una respuesta al problema del pasaje del silencio a la palabra en la filosofía de Merleau-Ponty. Areté. Revista de Filosofía, XXIX(2), 333-360. doi: doi.org/10.18800/arete.201702.005.

Buceta M. (2018a). Las ideas sensibles entre Merleau-Ponty y Proust. Diálogos, 103, 123-148.

Buceta M. (2018b). Merleau-Ponty y el problema de la verdad: la historia del pequeño Gauss y la suma de los ángulos del triángulo. Eidos, 29, 225-248. doi: 10.14482/eidos.29.1052.

Carbone, M. (2008). Proust et les idées sensibles. París: Vrin.

Carbone, M. (2012). Las ideas sensibles, entre vida y filosofía. En: M. T. Ramírez (Ed.), Merleau-Ponty viviente (pp. 99-111). Barcelona: Anthropos.

Carbone, M. (2015). Una deformación sin precedentes. Marcel Proust y las ideas sensibles. Barcelona: Anthropos.

42 "Scholarly discussions of Merleau-Ponty's aesthetics have tended to concentrate on his philosophy of painting [...] Comparatively less attention has been paid to his philosophy of literature." 
Collot, M. (1997). L'œuvre comme paysage d'une expérience. Merleau-Ponty et la critique thématique. En: S. A. Castin N. (Ed.), Merleau-Ponty et le littéraire (pp. 23-38). París: Rue d'Ulm.

Compagnon, A. (1987). Préface. En: M. Proust, Du côté de chez Swann (pp. 7-45). París: Gallimard.

Deleuze, G. (1964). Proust et les signes. París: Presses universitaires de France.

Descombes, V. (1987). Proust. Philosophie du roman. París: Ed. de Minuit.

Farrés Famadas, G. (2011). Merleau-Ponty, lector de Proust. Una presencia invisible. Paideia, 30(90), 79-93.

Husserl, E. (1950). Cartesianische Meditationen und Pariser Vorträge, Husserliana (Vol. I): Den Haag: Nijhoff.

Kearney, R. (2014). Écrire la chair : L'expression diacritique chez Merleau-Ponty. Chiasmi International, 15, 183-198. doi: 10.5840/chiasmi20131517

López Sáenz, M. C. (2008). Pensar filosóficamente, pensar literariamente. Merleau-Ponty y Proust. Investigaciones fenomenológicas. Anuario de la Sociedad Española de Fenomenología [Serie monográfica], 1, 307-341. doi: 10.5944/rif.1.2008.5568

Merleau-Ponty, M. (1945). Phénoménologie de la perception. París: Gallimard.

Merleau-Ponty, M. (1948). Sens et non-sens. París: Nagel.

Merleau-Ponty, M. (1953-1954). Le problème de la parole : Cours au Collège de France 1953-1954. Manuscrito inédito, Bibliothèque nationale de France, París, Francia.

Merleau-Ponty, M. (1960). Signes. París: Gallimard.

Merleau-Ponty, M. (1964). Le visible et linvisible. París: Gallimard.

Merleau-Ponty, M. (1968). Résumés de cours. Collège de France 1952-1960. París: Gallimard.

Merleau-Ponty, M. (1969). La prose du monde. París: Gallimard.

Merleau-Ponty, M. (1996). Notes de cours au Collège de France 1958-1959 et 1960-1961. París: Gallimard.

Merleau-Ponty, M. (2000). Parcours deux 1951-1961. Lonrai: Verdier.

Merleau-Ponty, M. (2002). Causeries. París: Seuil.

Merleau-Ponty, M. (2003). L'institution dans l'histoire personnelle et publique. Le problème de la passivité, le sommeil, l'inconscient, la mémoire. Notes de Cours au Collège de France 1954-1955. París: Belin. 
Merleau-Ponty, M. (2011). Le monde sensible et le monde de l'expression. Cours au Collège de France. Notes 1953. Genève: Mêtis Presses Sàrl.

Merleau-Ponty, M. (2013). Recherches sur l'usage littéraire du langage. Cours au Collège de France. Notes 1953. Genève: Mêtis Presses Sàrl.

Neefs, J. (1997). Le style est vision (Merleau-Ponty et Claude Simon). En: A. Simone \& N. Castin (Eds.), Merleau-Ponty et le littéraire (pp. 117-131). Paris: Rue d'Ulm.

Robert, F. (2008). Écriture et vérité. Revue internationale de philosophie, 2(244), 149-166. doi: 10.3917/rip.244.0149.

Sabot, P. (2002). Philosophie et littérature. Approches et enjeux d'une question. París: Presses universitaires de France.

Simone, A., \& Castin, N. (Eds.). (1997). Merleau-Ponty et le littéraire. París: Rue d'Ulm.

Waldenfels, B. (1998). Le paradoxe de l'expression chez Merleau-Ponty. En: M. Merleau-Ponty \& R. Barbaras, Notes de cours sur L’origine de la géométrie de Husserl; suivi de Recherches sur la phénoménologie de Merleau-Ponty (pp. 331-348). París: Presses universitaires de France. 